\title{
Time dependence of the helium flux measured by PAMELA
}

N. Marcelli ${ }^{1,20, a}$, O. Adriani ${ }^{2,3}$, G. C. Barbarino ${ }^{4,5}$, G. A. Bazilevskaya ${ }^{6}$, R. Bellotti $^{7,8}$, M. Boezio $^{9}$, E. A. Bogomolov ${ }^{10}$, M. Bongi ${ }^{2,3}$, V. Bonvicini ${ }^{9}$, S. Bottai ${ }^{3}$, A. Bruno ${ }^{8}$, F. Cafagna ${ }^{7,8}$, D. Campana ${ }^{5}$, P. Carlson ${ }^{11}$, M. Casolino ${ }^{1,12}$, G. Castellini ${ }^{13}$, C. De Santis ${ }^{1}$, V. Di Felice ${ }^{1,14}$, A. M. Galper ${ }^{15}$, A. V. Karelin ${ }^{15}$, S. V. Koldashov ${ }^{15}$, S. Koldobskiy ${ }^{15}$, S. Y. Krutkov ${ }^{10}$, A. N. Kvashnin ${ }^{6}$, A. Leonov ${ }^{15}$, V. Malakhov ${ }^{15}$, L. Marcelli ${ }^{1}$, M. Martucci ${ }^{1,20}$, A. G. Mayorov ${ }^{15}$, W. Menn ${ }^{17}$, M. Mergè ${ }^{1,20}$, V. V. Mikhailov $^{15}$, E. Mocchiutti ${ }^{9}$, A. Monaco ${ }^{7,8}$, N. Mori ${ }^{3}$, R. Munini ${ }^{9}$, G. Osteria ${ }^{5}$, B. Panico ${ }^{5}$, P. Papini $^{3}$, M. Pearce ${ }^{11}$, P. Picozza ${ }^{1,20}$, M. Ricci ${ }^{16}$, S. B. Ricciarini ${ }^{13}$, M. Simon ${ }^{17}$, R. Sparvoli ${ }^{1,20}$, P. Spillantini ${ }^{2,3}$, Y. I. Stozhkov ${ }^{6}$, A. Vacchi ${ }^{9,19}$, E. Vannuccini ${ }^{3}$, G. Vasilyev ${ }^{10}$, S. A. Voronov ${ }^{15}$, Y. T. Yurkin $^{15}$, G. Zampa ${ }^{9}$, N. Zampa ${ }^{9}$, M. S. Potgieter ${ }^{21}$, O. P. M. Aslam²1, and D. Bisschoff ${ }^{21}$

1 INFN, Sezione di Rome "Tor Vergata", I-00133 Rome, Italy

${ }^{2}$ University of Florence, Department of Physics, I-50019 Sesto Fiorentino, Florence, Italy

${ }^{3}$ INFN, Sezione di Florence, I-50019 Sesto Fiorentino, Florence, Italy

${ }^{4}$ University of Naples "Federico II", Department of Physics, I-80126 Naples, Italy

${ }^{5}$ INFN,Sezione di Naples, I-80126 Naples, Italy

${ }^{6}$ Lebedev Physical Institute, RU-119991, Moscow, Russia

${ }^{7}$ University of Bari, Department of Physics, I-70126 Bari, Italy

${ }^{8}$ INFN, Sezione di Bari, I-70126 Bari, Italy

${ }^{9}$ INFN, Sezione di Trieste, I-34149 Trieste, Italy

${ }^{10}$ Ioffe Physical Technical Institute, RU-194021 St. Petersburg, Russia

${ }^{11} \mathrm{KTH}$, Department of Physics, and the Oskar Klein Centre for Cosmoparticle Physics, AlbaNova University Centre, SE-10691 Stockholm, Sweden

${ }^{12}$ RIKEN, EUSO team Global Research Cluster, Wako-shi, Saitama, Japan

${ }^{13}$ IFAC, I-50019 Sesto Fiorentino, Florence, Italy

${ }^{14}$ Space Science Data Center - Agenzia Spaziale Italiana, via del Politecnico, s.n.c., I-00133, Roma, Italy

${ }^{15}$ National Research Nuclear University MEPhl, RU-115409, Moscow, Russia

${ }^{16}$ INFN, Laboratori Nazionali di Frascati, Via Enrico Fermi 40, I-00044 Frascati, Italy

${ }^{17}$ Universitat Siegen, Department of Physics, D-57068 Siegen, Germany

${ }^{18}$ Istituto Nazionale di Astrofisica, Fosso del cavaliere 100, 00133 Roma, Italy

${ }^{19}$ University of Udine, Department of Mathematics, Computer Science and Physics Via delle Scienze, 206, Udine, Italy

${ }^{20}$ University of Rome "Tor Vergata", Department of Physics, I-00133 Rome, Italy

${ }^{21}$ North-West University, Centre for Space Research, 2520 Potchefstroom, South Africa

\begin{abstract}
Precision measurements of the $Z=2$ component in cosmic radiation provide crucial information about the origin and propagation of the second most abundant cosmic ray species in the Galaxy ( $9 \%$ of the total). These measurements, acquired with the PAMELA space experiment orbiting Earth, allow to study solar modulation in details. Helium modulation is compared to the modulation of protons to study possible dependencies on charge and mass. The time dependence of helium fluxes on a monthly basis
\end{abstract}

\footnotetext{
ae-mail: nadir.marcelli@roma2.infn.it
} 
measured by PAMELA has been studied for the period between July 2006 to January 2016 in the energy range from $800 \mathrm{MeV} / n$ to $\sim 20 \mathrm{GeV} / n$.

\section{Introduction}

Measurements of absolute fluxes and spectral shapes of primary cosmic rays are extremely important for better understanding both origin and propagation mechanisms of galactic cosmic rays (GCRs). During their propagation through the InterStellar Medium (ISM) from the source, cosmic rays lose energy, modifying their spectra and composition. Subsequently, by entering the heliosphere, GCRs are scattered by irregularities in the heliospheric magnetic field (HMF). They are transported by the expanding solar wind from the Sun to the outermost regions of the heliosphere and processes such as convection, diffusion, drifts and adiabatic deceleration become more important; the sum of these effects is known as solar modulation $[1,2]$.

At energies above $\sim 30 \mathrm{GeV}$ for protons and $\sim 15 \mathrm{GeV} / n$ for helium, solar modulation has a negligible influence on the observed spectra and intensity profiles which represent the ones measured beyond the heliopause $[2,3]$.

The solar activity possesses an approximately 11-year periodicity which adds to a 22-year periodicity associated to the solar magnetic field rearrangement and reversal that usually takes place around the time of solar maximum and reaches peak strength at the solar minimum. The record-setting minimum of activity of cycle $23^{r d}$ (2006-late 2009) [4] caused extremely unusual minimum modulation conditions for GCRs and this translated into a perfect environment to study the various processes that affect particles inside the heliosphere. After 2009, solar activity started to rise slowly up to January 2014 , however this was lower than the previous one and the $24^{\text {th }}$ solar cycle has been classified as one of the weakest in recent years in terms of e.g. sunspot number, solar wind speed and number of solar events [5-7].

During its almost ten years of collecting data, PAMELA was well suited to measure the impact on solar modulation on many cosmic ray species, due to its high redundant detectors and high precision measurements which permitted to collect unprecedented volume of data. PAMELA collaboration published already the data on observed modulation for species like protons [8], [9], electrons [10] and the $e^{-} / e^{+}$ratio [11].

\section{The instrument}

PAMELA (A Payload for Antimatter-Matter Exploration and Light-nuclei Astrophysics) is a satelliteborne experiment designed to make long duration observations of the cosmic radiation from Low Earth Orbit [12]. The instrument collected GCRs for almost 10 years from 2006 June 15 when it was launched from the Baikonur cosmodrome in Kazakhstan, up to January of 2016. PAMELA orbit was elliptical with altitudes ranging between 350 and $610 \mathrm{~km}$ with an inclination of $70^{\circ}$. After 2010 the orbit was changed to a circular one at a constant altitude of about $500 \mathrm{~km}$. The payload comprises a number of highly redundant detectors capable of identifying particles by providing charge, rigidity and velocity measurements over a very wide energy range. Multiple sub-detectors were built around a magnetic spectrometer, composed of a silicon tracking system [13] placed inside a $0.43 \mathrm{~T}$ permanent magnet. A system of six layers of plastic scintillators, arranged in three double planes (S1, S2, and S3), provides a fast signal for triggering the acquired data, particle identification measuring the ionization energy loss and the time of flight $(\mathrm{ToF})$ of particles passing through. An electromagnetic imaging $\mathrm{W} / \mathrm{Si}$ calorimeter (16.3 radiation lengths and 0.6 interaction lengths deep) provides hadronlepton discrimination [14]. A neutron counter [15] contributes to discrimination power by detecting 
the increased neutron production in the calorimeter associated with hadronic showers compared to electromagnetic ones, while a plastic scintillator, placed beneath the calorimeter, increases the identification of high-energy electrons. The whole apparatus is surrounded by an anti-coincidence system (AC) of three scintillators (CARD, CAS, and CAT) for the rejection of background events [16]. A comprehensive description of the instrument and main scientific results can be found in [17], [18]. The payload is schematically shown in Figure 1 left.

\section{Event selection}

Clean helium candidates are selected requiring a single track in the spectrometer and fully contained inside the instrumental acceptance. The tracker selection criteria require a lever-arm of at least 4 silicon planes and a minimum of 3 hit on both $\mathrm{X}$ and $\mathrm{Y}$ view. Down going particles are selected with time information measured by the Time of Flight (ToF) system. GCR helium nuclei was selected considering only particles with a rigidity greater by a factor 1.3 than the vertical cutoff, evaluated using the Störmer approximation, at a given orbital position of the satellite. Then the ionization energy loss over the silicon planes of the tracking system is used to identify the helium nuclei (see Figure 1 right). Finally the helium fluxes have been estimated taking in to account the livetime, acceptance and efficiencies of the instrument.
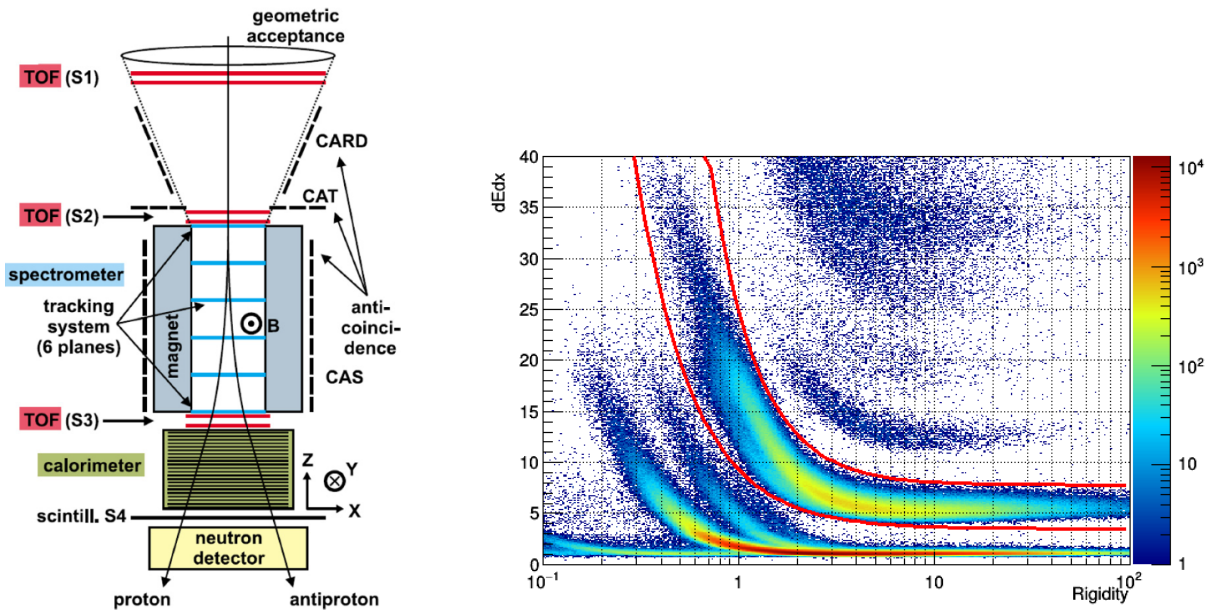

Figure 1. On the left: a schematic view of the PAMELA detector [17], [18]. On the right plot: the ionization energy loss distribution measured by the tracker as a function of rigidity. The region inside the two red curves represents the helium band considered for the selection criteria.

\section{Conclusions}

The time dependence of the helium fluxes measured by PAMELA offer the possibility to study the solar modulation of GCRs. The helium fluxes have been computed for a time period of one Carrington Rotation between July 2006 and January 2016, in the energy range from $800 \mathrm{MeV} / n$ and $\sim 20 \mathrm{GeV} / \mathrm{n}$; the results of this analysis will be published soon. Furthermore, comparison of protons and helium fluxes, through the time evolution of the $\mathrm{p} / \mathrm{He}$ ratio in the overall period of PAMELA data-taking, offer the possibility to study possible differences in modulation between these two main CR species. 


\section{References}

[1] E.N. Parker, Planetary and Space Science 13, 9 (1965)

[2] M.S. Potgieter, Space Sci. Rev.176, 165 (2013)

[3] R.D. Strauss, M.S. Potgieter, Advances in Space Research 53, 1015 (2014)

[4] R.A. Mewaldt, A.J. Davis, K.A. Lave, R.A. Leske, E.C. Stone, M.E. Wiedenbeck, W.R. Binns, E.R. Christian, A.C. Cummings, G.A. de Nolfo et al., Astrophys. J. Lett.723, L1 (2010)

[5] K.P. Schröder, M. Mittag, J.H.M.M. Schmitt, D. Jack, A. Hempelmann, J.N. González-Pérez, 470, 276 (2017), 1705.03777

[6] O.P.M. Aslam, Badruddin, Solar Phys.290, 2333 (2015), 1507.07917

[7] J. Jiang, R.H. Cameron, M. Schüssler, Astrophys. J. Lett.808, L28 (2015), 1507.01764

[8] O. Adriani, G.C. Barbarino, G.A. Bazilevskaya, R. Bellotti, M. Boezio, E.A. Bogomolov, M. Bongi, V. Bonvicini, S. Borisov, S. Bottai et al., ApJ 765, 91 (2013), 1301.4108

[9] M. Martucci, R. Munini, M. Boezio, V. Di Felice, O. Adriani, G.C. Barbarino, G.A. Bazilevskaya, R. Bellotti, M. Bongi, V. Bonvicini et al., Astrophys. J. Lett.854, L2 (2018), 1801.07112

[10] O. Adriani, G.C. Barbarino, G.A. Bazilevskaya, R. Bellotti, M. Boezio, E.A. Bogomolov, M. Bongi, V. Bonvicini, S. Bottai, A. Bruno et al., ApJ 810, 142 (2015), 1512 .01079

[11] O. Adriani, G.C. Barbarino, G.A. Bazilevskaya, R. Bellotti, M. Boezio, E.A. Bogomolov, M. Bongi, V. Bonvicini, S. Bottai, A. Bruno et al., Physical Review Letters 116, 241105 (2016), 1606.08626

[12] P. Picozza, A.M. Galper, G. Castellini, O. Adriani, F. Altamura, M. Ambriola, G.C. Barbarino, A. Basili, G.A. Bazilevskaja, R. Bencardino et al., Astroparticle Physics 27, 296 (2007), astro-ph/0608697

[13] O. Adriani, L. Bonechi, M. Bongi, G. Castellini, R. D’Alessandro, A. Gabbanini, M. Grandi, P. Papini, S.B. Ricciarini, P. Spillantini et al., Nuclear Instruments and Methods in Physics Research A 511, 72 (2003)

[14] M. Boezio, V. Bonvicini, E. Mocchiutti, P. Schiavon, G. Scian, A. Vacchi, G. Zampa, N. Zampa, Nuclear Instruments and Methods in Physics Research A 487, 407 (2002), physics/0109010

[15] Y.I. Stozhkov, A. Basili, R. Bencardino, M. Casolino, M.P. de Pascale, G. Furano, A. Menicucci, M. Minori, A. Morselli, P. Picozza et al., International Journal of Modern Physics A 20, 6745 (2005)

[16] S. Orsi, P. Carlson, P. Hofverberg, J. Lund, M. Pearce, International Cosmic Ray Conference 3, 369 (2005)

[17] O. Adriani, G.C. Barbarino, G.A. Bazilevskaya, R. Bellotti, M. Boezio, E.A. Bogomolov, M. Bongi, V. Bonvicini, S. Bottai, A. Bruno et al., Phys. Rep.544, 323 (2014)

[18] O. Adriani, G. Barbarino, G. Bazilevskaya, R. Bellotti, M. Boezio, E. Bogomolov, M. Bongi, V. Bonvicini, S. Bottai, A. Bruno et al., Rivista del Nuovo Cimento 40, 473 (2017) 